\title{
Principios organizativos del conocimiento en un programa de enfermería ${ }^{1}$
}

\author{
Alix Yaneth Perdomo Romero ${ }^{2}$ \\ Claudia Andrea Ramírez Perdomo ${ }^{3}$ \\ Dolly Arias Torres ${ }^{4}$
}

Institución: Universidad Surcolombiana

\section{CÓMO CITAR}

Perdomo, A., Ramírez, C. y Arias, D. (April, 2014). Principios organizativos del conocimiento en un programa de enfermería. Rev. Actual de Costa Rica, 26, 1-13. Disponible en: <http://www.revenf.ucr.ac.cr/conocimiento.pdf> ISSN 1409-4568

\section{RESUMEN}

Este artículo presenta los resultados de una investigación cuyo objetivo fue describir los principios organizativos del conocimiento en la formación de profesionales en enfermería surcolombianos. El estudio es cuali-cuantitativo, de corte transversal y descriptivo. La población la integraron 20 profesores y 80 estudiantes. La recolección de datos se efectuó a través de encuesta y entrevista semiestructurada. Los resultados demuestran que la estructura curricular del Programa de Enfermería es disciplinar, academicista, con predominio de un modelo asignaturista, con jerarquización de cursos; duplicidad de contenidos, trabajo fragmentado y desarticulación entre la teoría y la práctica. No se constatan ejes de integración, tampoco la interdisciplinariedad. Se concluye que se debe superar el prototipo disciplinar y la continuidad del currículo tradicional asignaturista, así como que la interdisciplinariedad garantiza que se potencialicen las fortalezas del currículo y la integración de los referentes teóricos y metodológicos para evitar la fragmentación del conocimiento.

Palabras clave: currículo, educación-en-enfermería, principios-organizativos.

\footnotetext{
${ }^{1}$ Fecha de recepción: 10 de setiembre del 2013

Fecha de aceptación: 28 de febrero del 2014

${ }^{2}$ Enfermera. Magíster en educación, profundización en gestión, diseño y evaluación curricular. Magíster en Enfermería con énfasis en atención al adulto anciano. Universidad Surcolombiana. Docente asistente, Universidad Surcolombiana. Colombia-Huila. Correo electrónico: alixyaneth3@yahoo.es

${ }^{3}$ Enfermera. Doctoranda de la Universidad de Antioquia. Magíster en enfermería con énfasis en gerencia en servicios de salud y enfermería. Especialista en cuidado crítico. Universidad Surcolombiana de Neiva. Docente asistente, Universidad Surcolombiana. Colombia-Huila. Correo electrónico clauram1@,hotmail.com

${ }^{4}$ Enfermera. Doctora en ciencias de la salud, República de Cuba. Magíster en educación y desarrollo comunitario. Universidad Surcolombiana. Docente titular, Universidad Surcolombiana. Colombia-Huila. Correo electrónico: dolaria@usco.edu.co
} 


\section{Organizational principles of knowledge in a nursing program ${ }^{1}$}

Institution: Surcolombiana University

Alix Yaneth Perdomo Romero ${ }^{2}$ Claudia Andrea Ramírez Perdomo ${ }^{3}$

Dolly Arias Torres ${ }^{4}$

\section{CITED}

\section{RESUMEN}

This article presents the results of a study aimed at describing the organizational principles of knowledge in the training of professionals nursing surcolombianos. The study is qualitative and quantitative, cross- sectional and descriptive. The population was composed of 20 teachers and 80 students. Data collection was conducted through survey and semi-structured interview. The results demonstrate that the curricular structure of the Nursing Program is to discipline, academic, predominantly an asignaturista model with ranking courses, content duplication, and fragmented work gap between theory and practice. No integration axes are detected, nor interdisciplinarity. We conclude they must overcome the discipline prototype and continuity of the asignaturista traditional curriculum. The Interdisciplinary curriculum ensures that the strengths of the curriculum make an enhance and integrate theoretical framework and methodological to avoid the fragmentation of knowledge.

Keywords: curriculum, nursing-education, organizational-principles

\footnotetext{
${ }^{1}$ Date of receipt: september 10, 2013

Date of acceptance: february 28, 2014

${ }^{2}$ Nurse. Master of education, deepening management, curriculum design and evaluation. Masters in Nursing with an emphasis on older adult care. Surcolombiana University. Teaching assistant, University Surcolombiana. Colombia Huila. E mail: alixyaneth3@yahoo.es ${ }^{3}$ Nurse. PhD from the University of Antioquia. Master in Nursing with an emphasis in management in health services and nursing. Critical care specialist. Surcolombiana University of Neiva. Teaching assistant, University Surcolombiana. Colombia Huila. .E mail: clauram1@hotmail.com

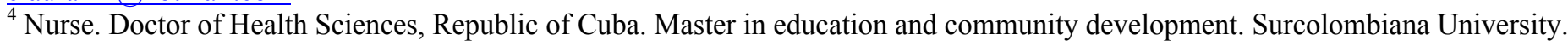
Full professor, University Surcolombiana. Colombia Huila. E mail: dolaria@usco.edu.co
} 


\section{INTRODUCCIÓN}

Según Bernstein (1985), el currículo se clasifica en dos tipos: el agregado, cuyos contenidos están claramente delimitados, aislados entre sí y con una relación cerrada, y el integrado, en los que la relación es abierta. Desde su teoría sociológica acerca de la organización y estructura del conocimiento, el autor plantea el interés de las instituciones en la búsqueda de un código integrado que no se opone al cambio.

Al respecto, Díaz (2002) señala que las instituciones de educación superior expresan interés de "transcender la tradicional organización académico-administrativa y curricular que ha hecho de las disciplinas y profesiones espacios aislados, desarticulados, carentes de reciprocidad e interdependencia; características propias de los modelos modernos de producción, de desarrollo del conocimiento y de la formación profesional" (p.21);sin embargo, la práctica dista de ese propósito.

En la revisión de literatura se encontraron investigaciones en enfermería, de las cuales Moya y Prado (2009), Moya y Esteban (2006) en España, concluyen que en la formación de enfermeros predomina un currículo instrumental y técnico, dirigido a la consecución de objetivos finales, caracterizado por la desarticulación existente entre el inicio de las ciencias básicas y la formación específica, lo mismo que el divorcio entre la teoría y la práctica. El aprendizaje es acumulativo y reproduccionista, "como si el conocimiento fuera fijo y acabado" (Moya, 2009, s.p.).

En México, Tejada, Castro, Miranda, Acosta y Alcántara (2008) analizaron lo concerniente al proceso de implementación de un plan de estudios reestructurado en forma radical,

con el propósito de formar un egresado que respondiera a las exigencias actuales, a los avances científicos, tecnológicos y sociales de la profesión y a resolver la falta de integración teórico práctica, desafío presente en numerosos programas de formación en Enfermería (p.81).

En sus resultados, los autores destacar la relevancia de enlazar la teoría con la práctica, del ingreso de pedagogías constructivistas y del logro de aprendizajes significativos para superar la transmisión de conocimiento; es decir, se prefiere un currículo integrado en el que el docente debe ser guía, apoyo, facilitador del aprendizaje y transformador de la racionalidad técnica en una reflexiva, mientras que el estudiante pasa a ser el centro de toda la acción.

En Brasil, Ramos y Calvacanti (2010) evidenciaron que, en la actualidad, el modelo tradicional se continúa a través del currículo disciplinar, fragmentado, el cual prevalece en la mayoría de las instituciones superiores. El plan de estudios formal, dividido en disciplinas, crea dificultades al estudiante en cuanto a articular el conocimiento fragmentado, razón por la que es necesario un cambio en la práctica de la enseñanza, de manera que el docente sea el mediador o facilitador del proceso de enseñanza-aprendizaje, en otras palabras, que sea quien despierte una práctica reflexiva en el estudiante para que desarrolle su autonomía y se convierta en un profesional que integre diversos saberes, partiendo del aprendizaje significativo.

En Colombia, Tovar y Villegas (2000) detectaron en los estudiantes de enfermería la necesidad de una participación activa, crítica y equitativa en la organización del currículo; en lo metodológico, por ejemplo, 


\section{Revista Electrónica Enfermería Actual en costa Rica}

mencionaron el predominio de la clase magistral, los pocos estudios de caso y propusieron mayor contacto con la práctica desde los primeros semestres.

Con base en los estudios referidos en párrafos precedentes se puede pensar que las estructuras académicas de los Programas Universitarios de Enfermería son academicistas, asignaturistas, atomizadas; al parecer, no hay relación entre teoría y práctica, persiste el conocimiento fragmentado, la inclusión de contenidos poco relevantes y no se visibiliza la investigación y la proyección social.

Dada la situación anterior, se consideró necesario desarrollar un análisis para describir los principios organizativos del conocimiento que orientan la formación del profesional de enfermería, ejercicio que puede sustentar el avance de un modelo pedagógico para el desarrollo de competencias técnico-científicas y de servicio social que requiere el mundo, el país y la región surcolombiana, cuya inspiración es la teoría del pensamiento complejo de Morin (1999), quien concibe la realidad como un todo en el que cualquier factor, por pequeño que parezca, puede afectar el comportamiento y la evolución. Al seguir dicho modelo, la educación en enfermería será pertinente y situada e induciría a modificar la actitud organizativa del conocimiento desde una perspectiva multidimensional; este accionar mantendrá vigente la formación de enfermeros con espíritu crítico, reflexivos y competentes para asumir los retos del mundo globalizado y del sistema de salud.

Sin obviar los principios del pensamiento complejo propuestos por Morín, a continuación se presentan dos enfoques relacionados con el currículo agregado e integrado propuesto por Bernstein (1985). En el primero, llamado asignaturismo, predominan los currículos de colección, el conocimiento disciplinar, insular, atomizado y fragmentado; el estudiante no descubre ni el sentido, ni la relevancia de lo aprendido.

En el segundo, el interdisciplinar, los currículos se integran, admiten la interacción de varias disciplinas, varios saberes, varios puntos de vista que se interconectan, lo cual permite identificar nexos entre las temáticas; promueve la apertura, el desarrollo cognitivo, el aprendizaje relacional, aparte de que forma un estudiante autónomo, crítico y reflexivo. Al respecto, López (2011) sostiene que uno de los retos de la educación superior es generar "cambios en los principios organizativos del conocimiento" (p.110); transformación que implica pasar de estructuras curriculares agregadas, yuxtapuestas, enciclopédicas a estructuras curriculares integradas, interdisciplinarias, abiertas, dialogantes, problematizadoras e investigativas. Igualmente, Díaz (2002) considera importante el análisis de

la oposición entre el conocimiento paradigmático de las disciplinas y las nuevas formas de su organización y producción; la lógica disciplinar ha sido el criterio fundamental de la organización del conocimiento en la escuela. Esto se observa con mayor énfasis en la universidad en donde los contenidos de aprendizaje se organizan a partir de componentes disciplinares recontextualizadas en asignaturas (p. 22).

Acerca de la temática, Young, citado por Díaz (Pedroza y García 2005), plantea que

la separación de las materias académicas de conocimiento relacionado con el trabajo se basa en dos premisas: la primera es que los primeros representan los objetivos de la educación de toda la persona y la segunda, la forma de conocimiento organizado, centrado en el rigor asociado a la 


\section{Revista Electrónica Enfermería Actual en costa Rica}

tradicional forma de existencia de las disciplinas y a las rivalidades académicas y profesionales que estas producen (p. 70).

\section{Mientras que Morin (2001) afirma que}

en todas partes se reconoce la necesidad de la interdisciplinariedad... que la rigidez de la lógica clásica sea corregida por una dialógica capaz de concebir nociones a la vez complementarias y antagónicas. La aptitud de contextualizar y de globalizar es una cualidad fundamental del espíritu humano, que la enseñanza parcelada atrofia y se debe, por el contrario, desarrollar un pensamiento capaz de relacionar, contextualizar y globalizar: un pensamiento complejo (p. 3).

Si bien es cierto la interdisciplinariedad busca la trasferencia de metodologías de una disciplina a otra, es necesario considerar el límite y el espacio de cada una para ratificar la identidad y autonomía profesional. De igual manera, hay que decir que dentro de los principios organizativos del conocimiento es preciso trabajar desde un enfoque sistémico, dialógico y de retroalimentación que promueva el desarrollo de una cultura académica a partir de modelos mentales que retomen el aprendizaje cooperativo y la responsabilidad social.

Respecto del asignaturismo, Euscátegui y Pino (2005) citan a López, quien expresa lo siguiente:

La actual estructura curricular es de colección, académica, enciclopédica y asignaturista; mantiene relaciones de verticalidad; el desempeño del docente es aislado y atomizado; el proceso de investigación no se constituye en un elemento central de la dinámica curricular y la evaluación y la reflexión permanentes están marginadas (p. 2).

Por otro lado, Iafrancesco (2004) manifiesta que de las investigaciones acerca de la integralidad de las experiencias de aprendizaje y la relevancia de las innovaciones se concluye que los problemas del aprendizaje acumulativo, constructivista y significativo no han recibido suficiente atención.

En síntesis se puede concluir que en un currículo agregado, asignaturista, con delimitación de contenidos y predominio de modelos pedagógicos instruccionales, orientado a la mecanización de los contenidos y procesos de evaluación memorísticos, se transmite un conocimiento con poco significado; su propósito central es transferir conocimientos, habilidades y destrezas de una disciplina o profesión y se convierte en obstáculo para buscar en el estudiante el aprendizaje significativo.

Finalmente, la interdisciplinariedad, mencionan Becerra (2009), Díaz (2002), López (2001), Iafrancesco (2004) facilita la introducción de nuevas estructuras curriculares con estrategias pedagógicas flexibles, sin jerarquías, que promuevan en el estudiante autonomía, responsabilidad, afectividad, motivación, creatividad, investigación, trabajo en equipo y pensamiento crítico; características que repercuten en el aprendizaje significativo. Los autores proponen enfoques pedagógicos interdisciplinarios aplicables en el área de la salud.

Acerca del concepto, $\underline{\text { Iafrancesco (2004) }}$ agrega que 
la interdisciplinariedad implica la colaboración entre las disciplinas, las ciencias, las artes, las tecnologías y la pedagogía; se opone al conocimiento fragmentado y se orienta a la integración y la globalización de los conocimientos y de la educación en general, lo cual implica la reestructuración de los planes de estudio de manera que permitan una visión global del saber y del saber hacer mediante la búsqueda sistemática de nexos e interrelaciones entre los distintos conocimientos, pero puestos, estos conocimientos, al servicio del desarrollo del ser humano y de la solución de los problemas de la comunidad (p. 35).

La interdisciplinariedad se construye a través de trabajo en equipo, cooperativo, dialogizante, en el que concurren varios saberes, cuyo fin es fortalecer el conocimiento y, así, solucionar los diversos problemas, satisfacer necesidades sociales y fortalecer el desarrollo humano y profesional.

\section{MATERIALES Y MÉTODOS}

La población fue integrada por 80 estudiantes de los cuatro últimos semestres con matrícula vigente y 20 docentes con vínculo laboral en el momento de recolectar de los datos. Dado el bajo tamaño de la población, se tomaron todos los elementos para el estudio.

Se diseñó una investigación cuali-cuantitativa, de corte transversal y descriptivo, desarrollada en el primer periodo académico de 2011.

En cuanto a los criterios de inclusión, los docentes debían tener un vínculo laboral en el momento de recolectar los datos, mientras que en el caso de los estudiantes, debían contar con matrícula vigente en el periodo 2011-A y haber cursado los últimos cuatro semestres. En la entrevista semiestructurada participaron 3 docentes y 5 estudiantes.

Para recabar información se utilizó un cuestionario constituido inicialmente por 32 ítems, cuyos enunciados se formularon según la escala tipo likert; luego, este fue sometido a juicio de expertos, quienes consideraron los siguientes criterios de evaluación: pertinencia, relevancia, claridad y coherencia: a partir de sus respuestas se determinó la validez de contenido y se mantuvieron solo 28 ítems. La consistencia interna se valoró mediante el Alfa de Cronbach, ejercicio con el que se ajustó el instrumento y se obtuvo una confiabilidad de 0,801 ; por tanto, la consistencia interna es alta.

La información recolectada se sistematizó y depuró en una hoja de cálculo de Microsoft Excel, versión 2007; después, la base de datos se pasó a un software estadístico, SPSS versión 15.0, con el que se calculó estadísticos descriptivos: distribución de frecuencias y porcentajes.

En cuanto al enfoque cualitativo, se aplicó una entrevista semiestructurada: a partir de una narración, los docentes y estudiantes expresaron sus sentimientos y percepciones, teniendo en cuenta la subjetividad, las experiencias y el significado que las personas tienen de sus vivencias, todo con el fin de describir los procesos organizativos del conocimiento que encierran la formación del profesional de enfermería y de conocer y analizar la realidad, 


\section{Revista Electrónica Enfermería Actual en costa Rica}

Las entrevistas fueron grabadas, transcritas en documento de microsoft word y luego impresas para codificar los datos, identificar categorías y su interrelación hasta clasificarlas en en asignaturismo e interdisciplinariedad.

\section{Consideraciones éticas}

Según la Resolución 8430 de Octubre de 1993 que establece normas científicas, técnicas y administrativas para la investigación en salud, este proyecto no contiene riesgos, dado que la recolección de datos no afectó la salud o integridad de las personas; aparte, durante su desarrollo prevaleció el criterio del respeto a la dignidad, la protección de los derechos y el bienestar de los sujetos de estudio y se garantizó la confidencialidad de los datos; los participantes tuvieron conocimiento de la naturaleza, de los procedimientos, beneficios y riesgos a que se someterían y la capacidad de libre elección; una vez que aceptaron participar, se les brindó el consentimiento informado, el cual contó con el aval del Comité de Ética de la Facultad de Salud.

\section{RESULTADOS}

De los participantes en la investigación, el $80 \%$ corresponde a estudiantes y el $20 \%$, a docentes. Las respuestas se agruparon en dos categorías: asignaturismo e interdisciplinariedad.

\section{Asignaturismo}

El 72\% de los encuestados está totalmente de acuerdo con que el currículo del Programa de Enfermería se encuentra distribuido por cursos y con prerrequisitos. El 19,5\% expresó estar totalmente de acuerdo con que falta integración entre las temáticas desarrolladas en cada curso y el 29,9\% está parcialmente de acuerdo. El 37,9\% y el $36,8 \%$ están parcialmente de acuerdo y totalmente de acuerdo en que los contenidos que se repiten durante el desarrollo de los diferentes cursos incrementa el esfuerzo de docentes y estudiantes: según ellos estos temas podrían establecer conexiones con otras disciplinas y favorecer el aprendizaje.

Acerca de los cursos de formación específica, el 35,6\% está parcialmente de acuerdo y el 29,9\% totalmente de acuerdo con que los contenidos de los cursos se desarrollan de forma desarticulada. También se detectó cierta tendencia a estar totalmente de acuerdo con la existencia de un currículo fundamentalmente presencial.

En cuanto a las características del currículo, los sujetos perciben mayor énfasis en contenidos, en vez de en prácticas pedagógicas, de lo que se deriva encasillamiento, desarticulación y abordaje de contenidos que no tienen aplicabilidad durante el desarrollo de la práctica; asimismo comentaron que hay poca comunicación entre los docentes de diferentes áreas y cursos, situación que aumenta la brecha entre teoría-práctica y redunda en un aprendizaje memorístico.

Las siguientes apreciaciones obtenidas a través de entrevistas reafirman el análisis descrito en párrafos precedentes: 
- "Uno de los problemas fundamentales en el Programa de Enfermería ha sido el encasillamiento de las asignaturas y el aislamiento de cada una; ejemplo, la morfología solo se dedica a la anatomía; la fisiología va por otro lado" (E1).

- "Falta conexión con el docente, hay temas que no tienen aplicabilidad en nuestra práctica profesional" (E3).

- "Cada área del saber o cada dominio específico lidera los contenidos desde su área; no hay articulación de disciplinas; esta es una debilidad grande" (D1).

- "Yo pienso que hay un problema: nosotros en el Ier semestre vemos la primera parte de morfo y físiología; simultáneamente, vemos bases conceptuales en enfermería, curso en el que poco se aplica lo abordado en morfofisiología. En el IIIer, IV y hasta casi V semestre, tenemos que volver a tomar lo que se vio en el Ier y II; en estos periodos ya hemos olvidado gran parte de lo visto en los dos primeros semestres" (E4).

- 'Nosotros vemos en el Ier y II semestre toda la anatomía del cuerpo humano, aún no entendemos qué es lo importante o esencial para nuestra práctica; en otras ocasiones profundizamos en cosas que no necesitamos" (E3).

- "Primero es la teoría y luego la práctica; se está fraccionado el conocimiento, cuando llegan a la práctica se ha olvidado la mitad" (E2).

- "Cuando uno avanza en los semestres se olvidan muchas cosas de los primeros cursos: hace falta más conexión entre ellos" (E5).

En síntesis, en el currículo de enfermería estudiado predomina el modelo asignaturista con jerarquización de cursos, duplicidad de contenidos, trabajo fragmentado y desarticulación entre la teoría y la práctica; aprendizaje memorístico y acumulativo y no se constatan ejes de integración ni de interdisciplinariedad.

\section{Interdisciplinariedad}

El 44,2\% está parcialmente de acuerdo con que el desarrollo de los cursos emplea la metodología basada en problemas, proyectos o núcleos temáticos y problemáticos; mientras que el $12,8 \%, 9,3 \%, 11,5 \%$ y $22,1 \%$ respectivamente, están totalmente de acuerdo, totalmente en desacuerdo, parcialmente en desacuerdo, ni de acuerdo ni en desacuerdo. El 41,4\% están parcialmente de acuerdo con que el desarrollo de los cursos promueve el autoaprendizaje, entretanto el $36,6 \%$ está totalmente de acuerdo.

El 27,1\% está parcialmente en desacuerdo con la existencia de ejes de integración entre los cursos, el $12,9 \%, 34,1 \%$ y $10,6 \%$ respectivamente, están totalmente en desacuerdo, parcialmente de acuerdo y totalmente de acuerdo. 


\section{Revista Electrónica Enfermería Actual en costa Rica}

Un 40,2\% están totalmente de acuerdo y un 36,8\% parcialmente de acuerdo con que, durante el desarrollo de las prácticas clínicas y comunitarias, se trabaja de manera interdisciplinar; el 4,6\%, y el 6,9\% están total y parcialmente en desacuerdo.

En relación con el conocimiento de la carga académica del estudiante por parte de los docentes, el 42,5\%, $23,0 \%, 12,65,13,8 \%$ y $8,0 \%$ manifestaron respectivamente, estar totalmente en desacuerdo, parcialmente en desacuerdo, ni de acuerdo ni en desacuerdo, parcialmente de acuerdo y totalmente de acuerdo.

La información descrita en párrafos anteriores se confirma en las siguientes expresiones:

- "Es en la práctica que se aprecia la relación de los contenidos de los diversos cursos, por ejemplo: es en esta que se aplican los principios de la farmacología” (E1).

- "La interdisciplinariedad permite ampliar la cosmovisión frente a los procesos de salud enfermedad y de esta manera aporta más elementos para tomar decisiones acertadas” (D2).

- "Es difícil contrastar realidades con una sola mirada; la naturaleza humana es de lo más complejo y para abordarla en toda su dimensión se requieren varios puntos de vista; yo creo que hay que apuntarle a la interdisciplinariedad" (D3).

- "Si las temáticas se vieran al tiempo; es decir, el farmacólogo y el enfermero interactuaran, sería más provechoso, aprenderíamos más” (E2).

- "Morfofisiología debería verse en la medida en que se avanza en las enfermerías, para así gradualmente complementar los conocimientos" (E5).

\section{DISCUSIÓN}

El predominio del asignaturismo en este estudio coincide con los aportes de López (2003), quien afirma que,

existe una marcada rigidez entre los diferentes campos del saber y las asignaturas que lo conforman, creando yuxtaposición y duplicidad de acciones y contenidos, lo cual pone en evidencia que en la estructura curricular existe una gama de micropoderes que difícilmente ceden para favorecer el proceso de integración (p. 30).

Para Moya y Parra (2006), el currículum en la formación de enfermeras evidencia jerarquía en los niveles de conocimiento profesional, lo cual implica una secuencia "lógica" en el aprendizaje, en donde en primer lugar deben aprenderse las ciencias básicas; ese orden jerarquiza roles, mientras que los niveles más aplicados y cercanos a la práctica están subordinados a los más abstractos que producen el conocimiento: "El saber que producen las ciencias en las que se funda el conocimiento profesional de la enfermera, léase biológicas y psicológicas, es un conocimiento analítico, fragmentado $\mathrm{y}$, en ocasiones, poco relevante para la práctica de enfermería" (p.p. 307-308). 


\section{Revista Electrónica Enfermería Actual en costa Rica}

Respecto de lo anterior, la investigación de la Universidad Nacional de Colombia (Gómez et al. 2003) relacionada con los problemas curriculares y pedagógicos, concluye que

la organización monodisciplinaria de los planes de estudio es un importante obstáculo para la formación interdisciplinaria, necesaria en el ejercicio de toda actividad profesional. En esta situación incide de manera significativa el patrón vigente de organización y división del trabajo académico generado por las facultades actuales. Algunas son de carácter monodisciplinario y otras son solo entes administradores de diversas carreras monodisciplinarias, carentes de interacción y sinergias mutuas.

En la actualidad, según Lazo (2011),

la preocupación de muchos docentes es que sus estudiantes adquieran los conocimientos de las diferentes asignaturas sin preocuparse de la relación existente entre sus núcleos cognitivos, relación que permitiría al estudiante analizar de forma orgánica y sintética los fenómenos. Este hecho sigue ocurriendo a pesar de las investigaciones realizadas por diferentes pedagogos que se pronuncian a favor de la interdisciplinariedad como el camino que permite la formación de una concepción integral, dialéctica y científica del mundo, desarrollando en los estudiantes un pensamiento verdaderamente científico, creativo e innovador (párr. 22).

En el currículo del programa estudiado hay jerarquización, disgregación, insularidad y atomización; brecha la teoría y la práctica y de las asignaturas de las ciencias básicas y sociohumanística con las de formación específica:

Basar la práctica en un conocimiento que defiende la fragmentación analítica y la cuantificación supone ignorar que la realidad es holística, lo cual impide analizar los problemas en contexto o encontrar significados y explicaciones. En efecto, reducir las personas y las prácticas del cuidado a partes de un mecanismo estable, regular y, por tanto, predecible, o a datos que puedan ser objeto de operaciones matemáticas nunca ha sido congruente con los valores tradicionales y aceptados en enfermería: holismo y humanismo (Moya y Prado 2006 p. 308).

El currículo del programa de enfermería se ha centrado en asignaturas, fenómeno que prevalece en las actuales estructuras curriculares del sistema educativo. Al respecto, López (2003), afirma que "cuando la clasificación y la enmarcación son fuertes (barreras, límites y roles marcadamente definidos, como es el caso del sistema educativo colombiano), se presenta un tipo de currículo agregado o de colección" (p. 41), en el que predomina la cultura académica, insular, disgregada, atomizada, que fracciona el conocimiento, aísla los otros saberes y obstaculiza la formación integral e interdisciplinar.

La interdisciplinariedad se interpretó como la participación de varias disciplinas en el desarrollo de las prácticas clínicas y comunitarias, mezcla en la que se confundió el significado de integración, interrelación e intercambio de saberes que esta implica; en las respuestas de los sujetos de estudio se encontró poca coherencia entre la predominancia del asignaturismo y la interdisciplinariedad del currículo. 


\section{Revista Electrónica Enfermería Actual en costa Rica}

Según Eslava (2002), la interdisciplinariedad es la relación entre las diversas disciplinas, conexas entre sí, que articulan sus saberes para permitir "el análisis de los fenómenos y procesos como un todo" (p. 37). Desde esta perspectiva, la interdisciplinariedad contribuye a la formación integral y al diálogo de saberes para responder acertadamente a los cada vez más complejos problemas de la salud humana. Coinciden con estas consideraciones, Lazo (2011), quien expresa que su ausencia lleva a una estrechez mental que interfiere en la aplicación de soluciones integradoras a los problemas de un mundo globalizado, y Perera y Pérez, citados por Moner (2010), para quienes la preparación del futuro profesional debe incluir la interdisciplinariedad.

\section{CONCLUSIONES}

La estructura curricular del Programa de Enfermería es disciplinar, academicista, con predominio de un modelo asignaturista; se destaca la jerarquización de cursos; duplicidad de contenidos, trabajo fragmentado y desarticulación entre la teoría y la práctica. No se constatan ejes de integración, ni la interdisciplinariedad.

\section{REFERENCIAS BIBLIOGRÁFICAS}

Becerra, B. (2009). El Aprendizaje Basado en Problemas: su uso en áreas de ciencias de la salud en la educación superior. Reflexiones sobre Educación Universitaria IV. Bogotá: Universidad Nacional de Colombia. Facultad de Medicina.

Bernstein, B. (1985). Clasificación y enmarcación del conocimiento educativo. Revista electrónica Colombiana de Educación, (15), 31-52. Recuperado de http://es.scribd.com/doc/53283734/Revista-Colombiana-de-Educacion$\underline{\text { Bernstein }}$

Díaz, M. (Ed.). (2002). Flexibilidad y Educación Superior en Colombia. Bogotá: Instituto Colombiano para la Evaluación de la Educación (ICFES).

Eslava, D. (2002). Interdisciplinariedad, enfermería, comunidad y transdisciplinariedad: algunas notas para la reflexión. Investigación en Enfermería Imagen y Desarrollo, 4, 34-39.

Euscátegui, R., Pino, S. (2005) La reestructuración curricular como resultado de los procesos sociales. Revista Electrónica de la Red de Investigación Educativa, 1 (3) Recuperado de http://revista.iered.org

Gómez, V., Celis, J., Guatame, P., Díaz, O., Urbano, N., Téllez, O. et al. (2003). Problemas curriculares y pedagógicos del pregrado en la Universidad Nacional de Colombia. [sede Web].. Recuperada en http://www.agro.unalmed.edu.co/v2/tmp/reforma/docs/problemas curriculares pregrado un.pdf

Iafrancesco, G. (Ed.) (2004). Currículo y plan de estudios: estructura y planteamiento. Colombia: Editorial Delfín Ltda.

Lazo, M. (2011). La interdisciplinariedad y la integralidad una necesidad de los profesionales de la educación. Enciclopedia Virtual eumed.net, 3(27) Recuperada en http://www.eumed.net/rev/ced/27/malp.htm 


\section{Revista Electrónica Enfermería Actual en costa Rica}

López, N. (Ed.) (2001). La deconstrucción curricular. Bogotá: Editorial Magisterio.

López, N., (Ed) (2003). Retos para la construcción curricular: de la certeza al paradigma de la incertidumbre creativa. Bogotá: Editorial Magisterio.

López, N., Puentes A. (2011). Modernización curricular de la Universidad Surcolombiana: integración e interdisciplinariedad. Revista Entornos, 24, 103-122.

Moner, M., Cabalé, M., Fernández, M. Oramas Y. (2010) Aproximación a la interdisciplinariedad desde la Morfofisiología y la Enfermería. Revista Científica de la Universidad de Ciencias Médicas de Holguín, 14(3). Recuperado en http://www.cocmed.sld.cu/no143/pdf/no143ori01.pdf

Morín E. (1999). Los siete saberes necesarios para la educación del futuro. Barcelona: Paidós.

Morín, E. (2001). De la reforma universitaria. Revista Uni-pluri/versidad versión digital, Vol. 1 (2): [6]. Recuperado de http://aprendeenlinea.udea.edu.co/revistas/index.php/unip/article/viewFile/12260/11120

Moya J. y Esteban, M. (2006). La complejidad del cuidado y el cuidado de la complejidad: un tránsito pedagógico de los reduccionismos fragmentantes a las lógicas no líneales de la complejidad. Texto \& Contexto Enfermagem, 15(2): 312-319. Recuperado de http://www.scielo.br/scielo.php?script=sci arttext\&pid=S010407072006000200016\&lng=en. http://dx.doi.org/10.1590/S0104-07072006000200016.

Moya J., y Parra S. (2006). La enseñanza de la enfermería como una práctica reflexiva. Texto \& Contexto Enfermagem, 15(2): 303-311. Recuperado de http://www.scielo.br/scielo.php?script=sci_arttext\&pid=S010407072006000200015\&lng=en. http://dx.doi.org/10.1590/S0104-07072006000200015

Moya, J. y Prado, M. (2009). El currículum de enfermería como prototipo de tejné: racionalidad instrumental y tecnológica. Texto \& Contexto - Enfermagem, 18(4), 617-626. Recuperado de http://www.scielo.br/scielo.php?script=sci arttext\&pid=S0104-07072009000400002\&lng=en\&tlng=es. $\underline{10.1590 / \mathrm{S} 0104-07072009000400002}$

Pedroza, R. y García, B. (Ed.). (2005). Compiladores. Flexibilidad académica y curricular en las instituciones de educación superior. México: Editorial Miguel Ángel Porrúa.

Ramos, R., Cavalcanti G.S. (2010) A prática docente reflexiva baseada no currículo integrado: uma questão de competências. Revista Iberoamericana de Educación, Vol. 54, No. 2. Recuperado de http://www.rieoei.org/deloslectores/3507Cavalcanti.pdf

Tejada, L., Castro, J., Miranda, M., Acosta L., Alcántara, E. (2008). Evaluación cualitativa de un programa de formación de enfermeras. La perspectiva de los profesores y los estudiantes. Revista Investigación y Educación en Enfermería, XXVI(2): [8]. Recuperado de

http://redalyc.uaemex.mx/src/inicio/ArtPdfRed.jsp?iCve=105215278006 


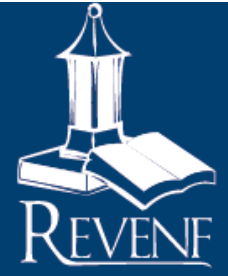

Tovar M., Villegas, L. (2000). Opiniones de los estudiantes de enfermería de la Universidad del Valle sobre los principios orientadores del nuevo currículo de enfermería. Revista Colombia Médica. 31(1): [28-36]. Recuperado de http://redalyc.uaemex.mx/redalyc/src/inicio/ArtPdfRed.jsp?iCve=28331107. 